\title{
Electronic Band Structure of Coiled Carbon Nanotubes
}

\author{
I. MiloševiĆ* And M. Damnjanović \\ NanoLab, Faculty of Physics, University of Belgrade, Studentski trg 12 \\ P.O. Box 44, Belgrade, 11001, Serbia
}

\begin{abstract}
More than fifteen years have passed since the first report of experimental evidence of regularly coiled carbon nanotubes, but, the structure, formation mechanism and theoretical aspects of these nanotubes still remain unresolved. We propose model of hexagonal, helically coiled single wall carbon nanotubes, determine their line group symmetry and calculate electronic band structure of the relaxed configurations by means of fully symmetry adopted density functional tight binding method implemented into the POLSym code. Electrical properties of the straight and coiled carbon nanotubes of different chiralities are compared and analyzed.
\end{abstract}

PACS: 61.48.De, 73.22.-f, 81.07.--b

\section{Introduction}

Coiled carbon nanotubes were observed experimentally in 1994 [1]. A yield of more than 95\% nanotubes grown by catalytic chemical vapor deposition have helical structure [2]. The coils have various diameters and pitches as each coil maintains its self-organization well during the growth. Thus far, synthesized coiled carbon nanotubes are multi-walled. Coiled single-walled nanotubes have been also observed, rarely though.

Several mechanisms for the formation of coiled carbon nanotubes were proposed: regular insertion of pentagonheptagon pairs at the junction [3], model of localized stresses and anisotropic rates of carbon deposition on catalyst particle [2] and thermodynamic model [4].

Morphology of helically coiled carbon nanotubes enables them to be used as high performance electromagnetic wave absorbers, sensors, resonators, mechanical springs, magnetic beam generators and electrical inductors. For applications it is needed to have control over the coil geometry. However, their structure, formation mechanism and theoretical aspects are still mysterious points [4].

In this article we propose a model of single-wall coiled carbon nanotubes, discuss their stability and calculate their electronic band structure.

\section{Morphology and Symmetry}

It was shown that coil formation in nanotubes comes out as a result of the competition between curvature elasticity and inter-layer adhesion [5], i.e. that curved deformation of the straight NTs can be analytically derived. Namely, it turned out that such an approach leads to

\footnotetext{
* corresponding author; e-mail: ivag@rcub.bg.ac.rs
}

equation of rigid string which has two exact solutions: straight line and regular coil. Accordingly, we propose a model of perfectly hexagonal helically coiled single wall carbon nanotubes, Fig. 1.

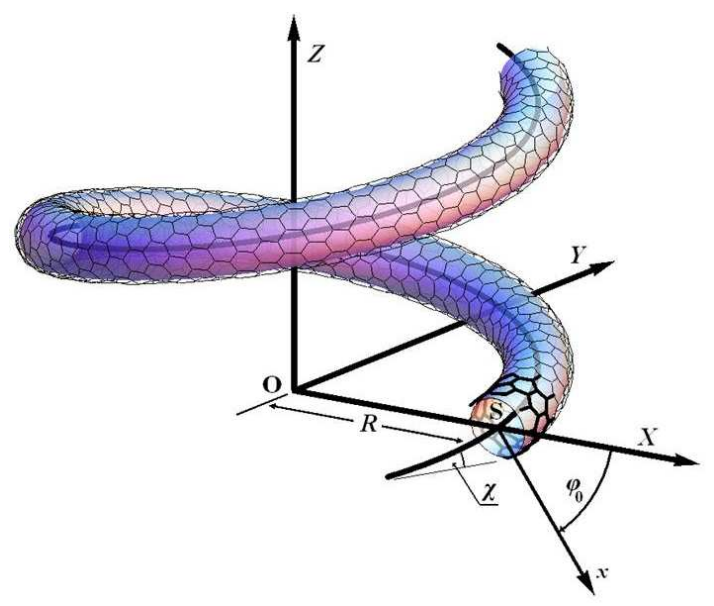

Fig. 1. Model of helically coiled carbon nanotube.

Technically, it is convenient to introduce coordinate system of a straight single-wall carbon nanotube $\left(n_{1}, n_{2}\right)$, in which $z$-axis is the tube axis, while $x$-axis is perpendicular to it and runs through the hexagon center [6]. Then helically coiled nanotube $\left(n_{1}, n_{2}\right)_{\left(R, \chi, \varphi_{0}\right)}$ is obtained as follows: Take a helix of radius $R$ around central (vertical) $Z$-axis and inclination angle $\chi$. Pull on it single-wall nanotube $\left(n_{1}, n_{2}\right)$ (homogeneously deforming it so that $z$-axis coincides with the helix). $X$-axis is defined as the axis perpendicular to the $Z$-axis and passes through the origin $S$ of the coordinate system of the coiled nanotube. Angle $\varphi_{0}$ is the angle formed by $X$ and $x$ axes and it describes rotation of the single-wall nanotube $\left(n_{1}, n_{2}\right)$ around the helix. 
Symmetries generated by helical transformations are given by Koster-Seitz elements $\left(C_{Q} \mid F\right)$ (rotations for $2 \pi / Q$ around $Z$-axis followed by translation for $F$ along $Z$-axis, $Q \geq 1$ and $F$ are to be determined) and form a line group [6] from the first line group family: $\boldsymbol{L}^{(1)}=$ $\boldsymbol{T}_{Q}(F) \boldsymbol{C}_{1}$. In a case of the special position when $\varphi_{0}=0$ there are also horizontal two-fold rotations (the so called $U$-axis which coincides with thew $X=x$ axis). Consequently, the coiled nanotube symmetry is twice larger and the relevant line group belongs to the fifth line group family: $\boldsymbol{L}^{(5)}=\boldsymbol{T}_{Q}(F) \boldsymbol{D}_{1}$.

In order to find $Q$ and $F$, it should be noted that a single translational period $a$ of a straight single-wall nanotube is the minimal part from which the coiled nanotube can be generated by applying the corresponding line group symmetry. Vertical and horizontal projections $F$ and $2 \pi R / Q$ of a unit cell vector a are:

$$
F=a \sin \chi, \quad Q=2 \pi R / a \cos \chi .
$$

Consequently, symcell [7] of a coiled nanotube contains $N=4 \frac{n_{1} n_{2}+n_{1}^{2}+n_{2}^{2}}{\operatorname{GCD}\left(2 n_{1}+n_{2}, n_{1}+2 n_{2}\right)}$ atoms (the same number of atoms as in the straight nanotube unit cell [7]).

Coiled nanotubes have translational symmetry only for rational values of $Q$, i.e. when $Q=q / r$ where $q$ and $r$ are coprime integers, $q \geq r$. In such a case the translational period is $A=q F$. Otherwise, helically coiled tube is incommensurate, with no translational periodicity whatsoever.

\section{Relaxation and Electronic Band Structure}

Above considerations shows that the coordinates of atoms of a perfect (without defects) helically coiled nanotube can be easily determined. Namely, arbitrary atom $P$ of the coiled tube of the helix radius $\tilde{\rho}$ is characterized by the coordinates $(\tilde{\varphi}, \tilde{z})$ : cross section of the helical tube through $P$ is circle with the center $S$, then $\tilde{z}$ is the length of the arc of the helix between $X Y$ plane and $S$, while $\tilde{\varphi}$ is angle between $S P$ and the (horizontal) direction perpendicular to $Z$ through $S$. Therefore, the coordinates $(\tilde{\rho}, \tilde{\varphi}, \tilde{z})$ of the atom of the helically coiled nanotube are the same as the cylindrical coordinates [7] $(\rho, \varphi, z)$ of the straight single-wall tube, depending only on the relative rotational position of the primary straight nanotube and of the helix, $\tilde{\varphi}=\varphi+\varphi_{0}$.

However, the Cartesian coordinates $(X, Y, Z)$ also depend on the helix parameters $R$ and $\chi$. Using $\zeta=\frac{\cos \chi}{R}$, elementary trigonometry gives:

$$
\begin{aligned}
X & =\left[\rho \cos \left(\tilde{\varphi}+\varphi_{0}\right)+R\right] \cos \zeta \tilde{z}-\rho \sin \left(\tilde{\varphi}+\varphi_{0}\right) \\
& \times \sin \chi \sin \zeta \tilde{z}, \\
Y & =\left[\rho \cos \left(\tilde{\varphi}+\varphi_{0}\right)+R\right] \sin \zeta \tilde{z}+\rho \sin \left(\tilde{\varphi}+\varphi_{0}\right) \\
& \times \sin \chi \cos \zeta \tilde{z} \\
Z & =\sin \chi \tilde{z}-\rho \cos \chi \sin \left(\tilde{\varphi}+\varphi_{0}\right) .
\end{aligned}
$$

Straight single-wall carbon nanotube $\left(n_{1}, n_{2}\right)$ can be generated from an arbitrary atom by the group action. Consequently, its translational period $a$, being single continuous parameter of its symmetry group, and coordi- nates of an atom determine the nanotube configuration. The coil itself is defined by the helix parameters $R$ and $\chi$. Finally, the angle $\varphi_{0}$ characterizes the position of the tube $\left(n_{1}, n_{2}\right)$ with respect to the helix. Hence, within described model, these seven parameters are sufficient to define the configuration of helically coiled nanotube.

Full line group symmetry is implemented in the energy band calculations through the modified group projector method. This is incorporated in the density functional tight-binding procedure within POLSym code [7], used to find relaxed configurations and electro-optical properties.

In the relaxation procedure we start with the initial configuration corresponding to the relaxed single-wall carbon nanotube coiled in a way that helix radius $R=5 r$ (where $r$ is radius of the straight nanotube), while the inclination angle $\chi$ gives helical step equal to $3 r$. Such a choice is a rough average of the shapes of the samples shown in literature [4]. The procedure turned up to converge, showing that there helically coiled configuration is sustainable, with parameters quite close to the initial ones.

Sample of fifty helically coiled carbon nanotubes with different chiral angles and diameters $(0.5 \mathrm{~nm}-1.2 \mathrm{~nm})$ is considered. Vast majority of the relaxed structures are found to have larger diameter and to be more tightly coiled than the initial, unrelaxed configuration, Fig. 2. However, there are also several exceptions to this.

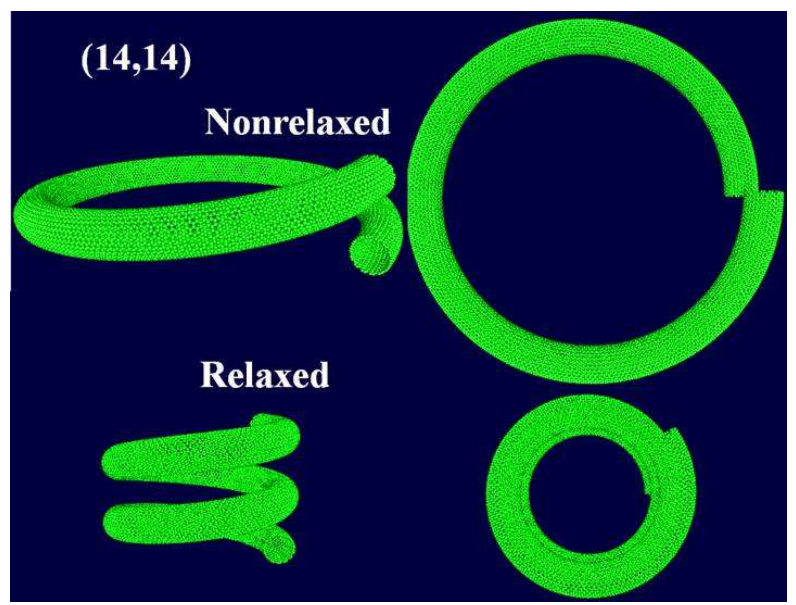

Fig. 2. Initial and relaxed configuration of helically coiled $(14,14)$ carbon nanotube.

Further, electronic band structure of the relaxed nanotubes have been calculated. Using POLSym code, the energies are assigned by the helical quantum number $\tilde{k}$ ( $\hbar \hat{k}$ is canonical momentum of the helical coordinate generated by the screw-axis $\boldsymbol{T}_{Q}(F)$ ) taking values from the helical Brillouin zone $(-\pi / F, \pi / F]$, and $\tilde{m}(\hbar \tilde{m}$ is the part of angular momentum which is not included in $\hbar \tilde{k}$ ), however, as rotational symmetry is trivial, angular momentum $\tilde{m}=0$, and the energies will be denoted as $E_{\tilde{m}}(\tilde{k})=E_{0}(\tilde{k})=E(\tilde{k})$. When $\varphi_{0}=0$, due to $U$-axis symmetry, the energies are double degenerate 
$E(\tilde{k})=E(-\tilde{k})$, and irreducible domain is $[0, \pi / F]$. Besides, there is parity quantum number $\Pi_{U}= \pm 1$ due to the $U$-axis symmetry and it characterizes electronic states at $\tilde{k}=0, \pi / F$.

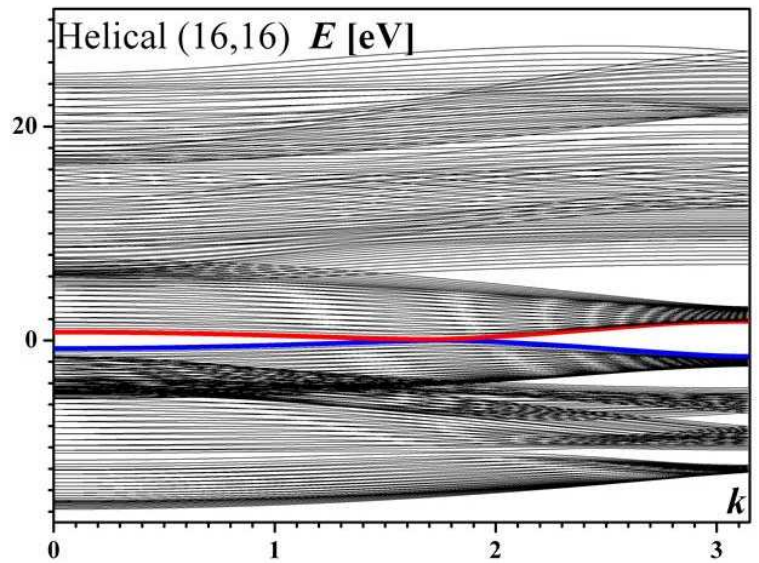

Fig. 3. Electronic energy bands of helically coiled $(16,16)$ carbon nanotube.

Electronic energy bands are calculated within tightbinding density functional method, with full symmetry implementing POLSym code [7]. Each atom contributed by four $s p^{3}$ orbitals. Roughly, electronic bands of the coiled nanotubes are similar to those of the corresponding straight nanotubes (Fig. 3) as far as the energy range and shape of the band structure as a whole are considered. Still, the number of bands of the coiled tubes is considerably larger since more orbitals per unit length is included. The main difference is that vast majority of the coils are conductive, with finite density of states at Fermi level. This can be interesting for applications.

\section{Conclusions}

It has been shown that thorough application of the full symmetry makes numerical calculations of the electronic band structure of the helically coiled carbon nanotubes feasible. Besides, introducing wave number which is conjugated to the helix rather than to the translational direction makes graphical presentation of the energy bands possible. The relaxation procedure proved to be effective and confirmed stability of the helically coiled structures. Vast majority of the coiled carbon nanotubes are found to be conductive with finite density of states at Fermi level. However, helically coiled armchair carbon nanotubes are shown to be narrow gap semiconducting as they loose their vertical mirror symmetry when coiled up and then Landau non-crossing rule becomes effective, Fig. 4.

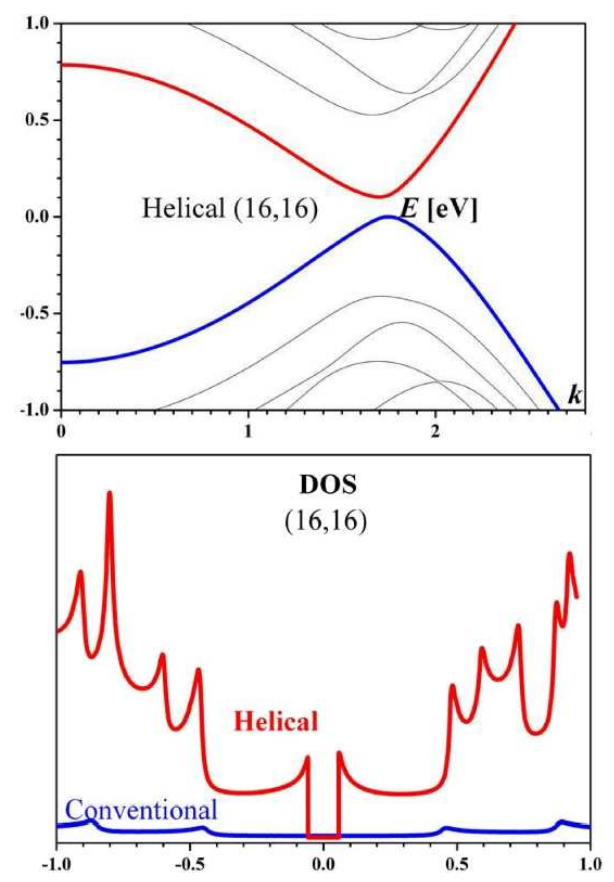

Fig. 4. Electronic energy bands and density of states of helically coiled $(16,16)$ carbon nanotube close to Fermi level.

\section{Acknowledgements}

The authors acknowledge funding of Serbian Ministry of Science (Project No. 171035) and Swiss National Science Foundation (SCOPES IZ73Z0-128037/1).

\section{References}

[1] S. Amelinckx, X.B. Zhang, D. Bernaerts, X.F. Zhang, V. Ivanov, J.B. Nagy, Science, 256, 635 (1994).

[2] X.B. Zhang, X.F. Zhang, D. Bernaerts, G. Van Tendeloo, S. Amelinckx, J. Van Landuyt, V. Ivanov, J.B. Nagy, Ph. Lambin, A.A. Lucas, Europhys. Lett. 27, 141 (1994).

[3] S. Itoh, S. Ihara, J. Kitakami, Phys. Rev. B 48, 1703 (1993).

[4] D. Fejes, K. Hernádi, Materials 3, 2618 (2010).

[5] O.-Y. Zhong-can, Z.-B. Zhao-Bin Su, C.-L. Wang, Phys. Rev. Lett. 78, 4055 (1997).

[6] M. Damnjanović, I. Milošević, Line Groups in Physics, Springer, Berlin 2010.

[7] M. Damnjanović, I. Milošević, E. Dobardžić, T. Vuković, B. Nikolić, in: Applied Physics of Nanotubes, Fundamentals of Theory, Optics and Transport Devices, Eds. S.V. Rotkin, S. Subramoney, Springer-Verlag, Berlin 2005, p. 41. 